\title{
Numerical simulations of stiff fluid gravitational singularities
}

\author{
Joshua Curtis \\ Department of Physics, University of Guelph, Guelph, Ontario, Canada N1G 2W1 \\ David Garfinkle* \\ Department of Physics, Oakland University, Rochester, MI 48309
}

\begin{abstract}
Numerical simulations of the approach to the singularity in spacetimes with stiff fluid matter are presented here. The spacetimes examined have no symmetries and can be regarded as representing the general behavior of singularities in the presence of such matter. It is found that the singularity is spacelike and that as it is approached, the spacetime dynamics becomes local and non-oscillatory.

PACS numbers: 04.20.Dw,04.25.Dm
\end{abstract}

\section{INTRODUCTION}

A longstanding problem in general relativity has been to find the general behavior of singularities. Several results, both analytical[1] and numerical [2] have been obtained. Though most of the results are for the case where the spacetimes have one or more symmetries, recent work has been done on the general case where there are no symmetries. [3, 4, 5] There is a longstanding conjecture 6] due to Belinski, Lifschitz and Khalatnikov (BKL) that states that the generic singularity is spacelike and local. This conjecture has been reformulated and put more precisely by Uggla et al. [7] The type of local dynamics conjectured by BKL depends on the type of matter. For vacuum and for many types of matter, the BKL conjecture is that the local dynamics is oscillatory, corresponding to the dynamics of a Bianchi type IX spacetime. However, for stiff fluid (i.e. fluid with pressure equal to energy density) the BKL conjecture is that the local dynamics is asymptotically velocity term dominated corresponding to the dynamics of a Bianchi type I spacetime. The vacuum version of the BKL conjecture has been supported by the numerical simulations of 5$]$ which show local and oscillatory dynamics in vacuum spacetimes with no symmetry. The stiff fluid version of the BKL conjecture has been supported by the theorem of Andersson and Rendall 3 ] which shows the local existence in a neighborhood of the singularity of solutions of the Einstein equations with stiff fluid matter with the expected asymptotic behavior and with enough degrees of freedom to be the generic solutions. What is not known is whether generic stiff fluid initial data evolves to a solution of the Andersson and Rendall class.

To address this issue, we perform numerical simulations of the approach to the singularity for stiff fluid matter with no symmetries. Our methods are those of $[5]$ using the system of [7]. Section II presents the equations and numerical methods used. The results are given in section III and conclusions in section IV.

*Electronic address: garfinkl@oakland.edu

\section{EQUATIONS}

The system evolved here is essentially that of reference 7] but specialized to the stiff fluid case and with a slightly different choice of gauge. Here the spacetime is described in terms of a coordinate system $\left(t, x^{i}\right)$ and a tetrad $\left(\mathbf{e}_{0}, \mathbf{e}_{\alpha}\right)$ where both the spatial coordinate index $i$ and the spatial tetrad index $\alpha$ go from 1 to 3 . It is assumed that $\mathbf{e}_{0}$ is hypersurface orthogonal and that the relation between tetrad and coordinates is of the form $\mathbf{e}_{0}=N^{-1} \partial_{t}$ and $\mathbf{e}_{\alpha}=e_{\alpha}{ }^{i} \partial_{i}$ Here $N$ is the lapse and we have chosen the shift to be zero. We choose the spatial frame $\left\{\mathbf{e}_{\alpha}\right\}$ to be Fermi propagated along the integral curves of $\mathbf{e}_{0}$. The commutators of the tetrad components are decomposed as follows:

$$
\begin{aligned}
{\left[\mathbf{e}_{0}, \mathbf{e}_{\alpha}\right] } & =\dot{u}_{\alpha} \mathbf{e}_{0}-\left(H \delta_{\alpha}{ }^{\beta}+\sigma_{\alpha}^{\beta}\right) \mathbf{e}_{\beta} \\
{\left[\mathbf{e}_{\alpha}, \mathbf{e}_{\beta}\right] } & =\left(2 a_{[\alpha} \delta_{\beta]}{ }^{\gamma}+\epsilon_{\alpha \beta \delta} n^{\delta \gamma}\right) \mathbf{e}_{\gamma}
\end{aligned}
$$

where $n^{\alpha \beta}$ is symmetric, and $\sigma^{\alpha \beta}$ is symmetric and trace free. Square brackets denote the antisymmetric part of a tensor.

Define $u^{a} \equiv e_{0}{ }^{a}$ and $h_{a b} \equiv g_{a b}+u_{a} u_{b}$, that is $u^{a}$ is the timelike vector of the tetrad and $h_{a b}$ is the spatial metric corresponding to the choice of $u^{a}$ as the time direction. Then the stress-energy tensor can be decomposed as

$$
T_{a b}=\mu u_{a} u_{b}+2 q_{(a} u_{b)}+p h_{a b}+\pi_{a b}
$$

where $q_{a}$ and $\pi_{a b}$ are orthogonal to $u^{a}$ and where $\pi_{a b}$ is symmetric and trace-free. Round brackets denote the symmetric part of a tensor.

Scale invariant variables are defined as follows:

$$
\begin{array}{r}
\left\{\boldsymbol{\partial}_{0}, \boldsymbol{\partial}_{\alpha}\right\} \equiv\left\{\mathbf{e}_{0}, \mathbf{e}_{\alpha}\right\} / H \\
\left\{E_{\alpha}{ }^{i}, \Sigma_{\alpha \beta}, A^{\alpha}, N_{\alpha \beta}\right\} \equiv\left\{e_{\alpha}{ }^{i}, \sigma_{\alpha \beta}, a^{\alpha}, n_{\alpha \beta}\right\} / H \\
q+1 \equiv-\boldsymbol{\partial}_{0} \ln H \\
r_{\alpha} \equiv-\boldsymbol{\partial}_{\alpha} \ln H \\
\left\{\Omega, P, Q^{\alpha}, \Pi_{\alpha \beta}\right\} \equiv\left\{\mu, p, q^{\alpha}, \pi_{\alpha \beta}\right\} /\left(3 H^{2}\right)
\end{array}
$$

The matter variables are not all independent, because we assume that the stress-energy is that of a stiff fluid

$$
T_{a b}=\tilde{\mu}\left(2 \tilde{u}_{a} \tilde{u}_{b}+g_{a b}\right)
$$


Here $\tilde{\mu}$ is the rest frame energy density of the fluid and $\tilde{u}^{a}$ is the fluid four-velocity, which can be decomposed as $\tilde{u}^{a}=\Gamma\left(u^{a}+v^{a}\right)$ where $v^{a}$ is orthogonal to $u^{a}$. Comparison of equations (3) and (9) yields

$$
\begin{array}{r}
Q_{\alpha}=\frac{2 \Omega}{G_{+}} v_{\alpha} \\
\Pi_{\alpha \beta}=\frac{2 \Omega}{G_{+}} v_{<\alpha} v_{\beta>} \\
P=\frac{\Omega}{G_{+}}\left(1-\frac{1}{3} v^{2}\right)
\end{array}
$$

Here $v^{2}=v^{\alpha} v_{\alpha}$ and $G_{+}=1+v^{2}$ and angle brackets denote the symmetric trace-free part of a tensor. Thus, all scale invariant matter variables can be expressed in terms of $\Omega$ and $v^{\alpha}$.

Finally choose the lapse to be $N=H^{-1}$. The relation between scale invariant frame derivatives and coordinate derivatives is $\boldsymbol{\partial}_{0}=\partial_{t}$ and $\boldsymbol{\partial}_{\alpha}=E_{\alpha}{ }^{i} \partial_{i}$. From the Einstein field equations and the conservation of stress-energy one obtains the following evolution equations:

$$
\begin{aligned}
\partial_{t} E_{\alpha}{ }^{i} & =F_{\alpha}{ }^{\beta} E_{\beta}{ }^{i} \\
\partial_{t} r_{\alpha} & =F_{\alpha}{ }^{\beta} r_{\beta}+\boldsymbol{\partial}_{\alpha} q \\
\partial_{t} A^{\alpha} & =F^{\alpha}{ }_{\beta} A^{\beta}+\frac{1}{2} \boldsymbol{\partial}_{\beta} \Sigma^{\alpha \beta} \\
\partial_{t} \Sigma^{\alpha \beta} & =(q-2) \Sigma^{\alpha \beta}-2 N^{<\alpha}{ }_{\gamma} N^{\beta>\gamma}+N_{\gamma}{ }^{\gamma} N^{<\alpha \beta>} \\
& +\partial^{<\alpha} r^{\beta>}-\boldsymbol{\partial}^{<\alpha} A^{\beta>}+2 r^{<\alpha} A^{\beta>} \\
& +\epsilon^{\gamma \delta<\alpha}\left(\boldsymbol{\partial}_{\gamma}-2 A_{\gamma}\right) N^{\beta>}{ }_{\delta}+3 \Pi^{\alpha \beta} \\
\partial_{t} N^{\alpha \beta} & =q N^{\alpha \beta}+2 \Sigma^{(\alpha}{ }_{\delta} N^{\beta) \delta}-\epsilon^{\gamma \delta(\alpha} \boldsymbol{\partial}_{\gamma} \Sigma^{\beta)}{ }_{\delta} \\
\partial_{t} \Omega & =(2 q-1) \Omega-3 P-\boldsymbol{\partial}_{\alpha} Q^{\alpha}+2 Q^{\alpha} A_{\alpha} \\
& -\Pi^{\alpha \beta} \Sigma_{\alpha \beta} \\
\partial_{t} v^{\alpha} & =\frac{G_{+}}{2 G_{-} \Omega}\left[\left(G_{-} \delta^{\alpha}{ }_{\beta}+2 v^{\alpha} v_{\beta}\right)\left(\partial_{t} Q^{\beta}-2[q+1] Q^{\beta}\right)\right. \\
& \left.-2 v^{\alpha}\left(\partial_{t} \Omega-2[q+1] \Omega\right)\right] \\
\partial_{t} q & =\left[2(q-2)+\frac{1}{3}\left(2 A^{\alpha}-r^{\alpha}\right) \boldsymbol{\partial}_{\alpha}-\frac{1}{3} \partial^{\alpha} \boldsymbol{\partial}_{\alpha}\right] q \\
& -\frac{4}{3} \boldsymbol{\partial}_{\alpha} r^{\alpha}+\frac{8}{3} A^{\alpha} r_{\alpha}+\frac{2}{3} r_{\beta} \boldsymbol{\partial}_{\alpha} \Sigma^{\alpha \beta}-2 \Sigma^{\alpha \beta} W_{\alpha \beta} \\
& +\frac{2}{G_{+}}\left[2 \Omega \Sigma^{\alpha \beta} v_{\alpha} v_{\beta}-2(q-2) \Omega+\partial_{t} \Omega\right. \\
& \left.-\frac{2 \Omega}{G_{+}} v_{\alpha} \partial_{t} v^{\alpha}\right]
\end{aligned}
$$

Here we are using units where $c=8 \pi G=1$. Furthermore the quantities $G_{-}, F_{\alpha \beta}, W_{\alpha \beta}$ and $\partial_{t} Q_{\alpha}$ are given by

$$
\begin{aligned}
G_{-} & \equiv 1-v^{2} \\
F_{\alpha \beta} & \equiv q \delta_{\alpha \beta}-\Sigma_{\alpha \beta} \\
W_{\alpha \beta} & \equiv \frac{2}{3} N_{\alpha \gamma} N_{\beta}^{\gamma}-\frac{1}{3} N_{\gamma}^{\gamma} N_{\alpha \beta}+\frac{1}{3} \boldsymbol{\partial}_{\alpha} A_{\beta} \\
& -\frac{2}{3} \boldsymbol{\partial}_{\alpha} r_{\beta}-\frac{1}{3} \epsilon_{\alpha}^{\gamma \delta}\left(\boldsymbol{\partial}_{\gamma}-2 A_{\gamma}\right) N_{\beta \delta} \\
\partial_{t} Q_{\alpha} & =2(q-1) Q_{\alpha}-\Sigma_{\alpha \beta} Q^{\beta}-\partial_{\alpha} P-\boldsymbol{\partial}^{\beta} \Pi_{\alpha \beta} \\
& +(P-\Omega) r_{\alpha}+\Pi_{\alpha \beta}\left(3 A^{\beta}+r^{\beta}\right) \\
& +\epsilon_{\alpha \beta \gamma} N^{\beta \delta} \Pi_{\delta} \gamma
\end{aligned}
$$

In addition to the evolution equations, the variables satisfy constraint equations as follows:

$$
\begin{aligned}
0 & =\left(\mathcal{C}_{\mathrm{com}}\right)^{\gamma i} \equiv \epsilon^{\alpha \beta \gamma}\left(\boldsymbol{\partial}_{\alpha} E_{\beta}{ }^{i}-\left[r_{\alpha}+A_{\alpha}\right] E_{\beta}{ }^{i}\right) \\
& -N^{\gamma \alpha} E_{\alpha}^{i} \\
0 & =\mathcal{C}_{\mathrm{G}} \equiv 1+\frac{1}{3}\left(2 \boldsymbol{\partial}_{\alpha}-2 r_{\alpha}-3 A_{\alpha}\right) A^{\alpha}-\frac{1}{6} N_{\alpha \beta} N^{\alpha \beta} \\
& +\frac{1}{12}\left(N^{\alpha}{ }_{\alpha}\right)^{2}-\frac{1}{6} \Sigma_{\alpha \beta} \Sigma^{\alpha \beta}-\Omega \\
0 & =\left(\mathcal{C}_{\mathrm{C}}\right)^{\alpha} \equiv \boldsymbol{\partial}_{\beta} \Sigma^{\alpha \beta}+2 r^{\alpha}-\Sigma^{\alpha}{ }_{\beta} r^{\beta}-3 A_{\beta} \Sigma^{\alpha \beta} \\
& -\epsilon^{\alpha \beta \gamma} N_{\beta \delta} \Sigma_{\gamma}{ }^{\delta}+3 Q^{\alpha} \\
0 & =\mathcal{C}_{q} \equiv q-\frac{1}{3} \Sigma^{\alpha \beta} \Sigma_{\alpha \beta}+\frac{1}{3} \partial_{\alpha} r^{\alpha}-\frac{2}{3} A_{\alpha} r^{\alpha} \\
& -\frac{1}{2}(\Omega+3 P) \\
0 & =\left(\mathcal{C}_{\mathrm{J}}\right)^{\alpha} \equiv \boldsymbol{\partial}_{\beta} N^{\alpha \beta}-\left(r_{\beta}+2 A_{\beta}\right) N^{\alpha \beta} \\
& +\epsilon^{\alpha \beta \gamma}\left(\boldsymbol{\partial}_{\beta} A_{\gamma}-r_{\beta} A_{\gamma}\right) \\
0 & =\left(\mathcal{C}_{\mathrm{W}}\right)^{\alpha}=\epsilon^{\alpha \beta \gamma}\left(\boldsymbol{\partial}_{\beta} r_{\gamma}-A_{\beta} r_{\gamma}\right)-N^{\alpha \beta} r_{\beta}
\end{aligned}
$$

We want a class of initial data satisfying these constraints that is general enough for our purposes but simple enough to find numerically. Recall that on an initial data surface, the spatial metric $h_{i j}$ and extrinsic curvature $K^{i j}$ must satisfy the constraint equations

$$
\begin{gathered}
D_{i}\left(K^{i j}-K h^{i j}\right)=q^{j} \\
R+K^{2}-K^{i j} K_{i j}=2 \mu
\end{gathered}
$$

Here $D_{i}$ and $R$ are respectively the derivative operator and scalar curvature associated with $h_{i j}$ and $\mu$ and $q_{i}$ are the components of the stress-energy tensor given in equation (3). We use the York method 8] which begins by defining the quantities $\bar{h}_{i j}$ and $\bar{A}^{i j}$ by

$$
\begin{array}{r}
h_{i j}=\psi^{4} \bar{h}_{i j} \\
K^{i j}-\frac{1}{3} K h^{i j}=\psi^{-10} \bar{A}^{i j}
\end{array}
$$

We choose $K$ to be constant, $\bar{h}_{i j}$ to be the flat metric $\delta_{i j}$ and $q^{i}$ to vanish. With these choices, equations (31) and (32) become

$$
\begin{aligned}
\partial_{i} \bar{A}^{i j} & =0 \\
\partial_{i} \partial^{i} \psi & =\left(\frac{1}{12} K^{2}-\frac{1}{4} \mu\right) \psi^{5}-\frac{1}{8} \bar{A}^{i j} \bar{A}_{i j} \psi^{-7}
\end{aligned}
$$

Here $\partial_{i}$ is the ordinary derivative with respect to Cartesian coordinates and indicies are raised and lowered with $\delta_{i j}$.

We choose space to have topology $T^{3}$ with the Cartesian coordinates $x, y$ and $z$ each going from 0 to $2 \pi$. We choose the following solution of equation (35)

$$
\begin{aligned}
\bar{A}^{11} & =a_{2} \cos y+a_{3} \cos z+b_{2}+b_{3} \\
\bar{A}^{22} & =a_{1} \cos x-a_{3} \cos z+b_{1}-b_{3} \\
\bar{A}^{33} & =-a_{1} \cos x-a_{2} \cos y-b_{1}-b_{2}
\end{aligned}
$$

with the off-diagonal components of $\bar{A}^{i j}$ vanishing. Here the $a_{i}$ and $b_{i}$ are constants. Note that due to the periodicity of the coordinates and the linearity of equation (35) 
the general solution of equation (35) is a Fourier series. The solution that we choose is then essentially the simplest solution of equation (35) without symmetries. The quantity $\mu$ can be freely specified and we choose it to be

$$
\mu=c_{0}+c_{1} \cos x+c_{2} \cos y+c_{3} \cos z
$$

where the $c_{i}$ are constants. With these choices for $\bar{A}^{i j}$ and $\mu$, equation (36) is solved numerically (in a manner to be described later) to yield $\psi$ and therefore $h_{i j}$ and $K^{i j}$.

From this initial data, we must then produce the initial values of the scale invariant variables. From equation (11) it follows that $H=-K / 3$ and since $H$ is constant it then follows that $r_{\alpha}$ vanishes. Since the initial spatial metric is conformally flat, we choose the initial spatial tetrad vectors by

$$
E_{\alpha}{ }^{i}=H^{-1} \psi^{-2} \delta_{\alpha}^{i}
$$

It then follows from equation (21) that $N_{\alpha \beta}$ vanishes and that

$$
A_{\alpha}=-2 \psi^{-1} \partial_{\alpha} \psi
$$

From equation (11) it then follows that

$$
\Sigma^{\alpha \beta}=-H^{-1} \psi^{-6} \delta^{\alpha}{ }_{i} \delta^{\beta}{ }_{j} \bar{A}^{i j}
$$

while $\Omega$ is given by equation (8) and $q$ by the vanishing of equation (28).

The numerical method used is as follows: each spatial direction corresponds to $n+2$ grid points with spacing $d x=2 \pi / n$. The variables on grid points 2 to $n+1$ are evolved using the evolution equations, while at points 1 and $n+2$ periodic boundary conditions are imposed. The initial data is determined once equation (36) is solved. This is done iteratively as follows: 9] Define

$$
S(\psi) \equiv-2 \psi+\left(\frac{1}{12} K^{2}-\frac{1}{4} \mu\right) \psi^{5}-\frac{1}{8} \bar{A}^{i j} \bar{A}_{i j} \psi^{-7}
$$

Then equation (36) takes the form $\partial^{i} \partial_{i} \psi-2 \psi=S(\psi)$. We make an initial guess $\psi^{0}$ for $\psi$ and solve using the conjugate gradient method 10 the equation

$$
\partial^{i} \partial_{i} \psi^{k+1}-2 \psi^{k+1}=S\left(\psi^{k}\right)
$$

iterating until $\psi^{k}$ satisfies equation (36) to within a set tolerance.

The evolution proceeds using equations (1320) with the exception that the term $(5-2 q) \mathcal{C}_{q}$ is added to the right hand side of equation (20) to prevent the growth of constraint violating modes and the term $-0.6\left(\mathcal{C}_{\mathrm{C}}\right)^{\alpha}$ is added to the right hand side of equation (15) to make the system well posed. 11 Spatial derivatives are evaluated using centered differences, and the evolution is done using a three step iterated Crank-Nicholson method 12] (a type of predictor-corrector method). In equation (20) the highest spatial derivative term is $-\frac{1}{3} \partial^{\alpha} \boldsymbol{\partial}_{\alpha} q$ which gives this equation the form of a diffusion equation. Note that diffusion equations can only be evolved in one direction in time, in this case the negative direction which corresponds to the approach to the singularity. Stability of numerical evolution of diffusion equations generally requires a time step proportional to the square of the spatial step. However, the constant of proportionality depends on the coefficient of the second spatial derivative. To ensure stability, we define $E_{\max }$ to be the maximum value of $\left|E_{\alpha}{ }^{i}\right|$ (over all space and over all $\alpha$ and $i$ ) and then define $d t_{1} \equiv-\frac{1}{4}\left(d x / E_{\max }\right)^{2}$ and $d t_{2} \equiv-\frac{1}{8} d x$. The time step $d t$ is then chosen to be whichever of $d t_{1}$ and $d t_{2}$ has the smaller magnitude.

Before presenting numerical results, it is helpful to consider what behavior to expect as the singularity is approached (that is as $t \rightarrow-\infty$ ). First denote the eigenvalues of $\Sigma^{\alpha}{ }_{\beta}$ by $\left(\Sigma_{1}, \Sigma_{2}, \Sigma_{3}\right)$. Then suppose that at sufficiently early times the time averages of $q-\Sigma_{i}$ are all positive. Then the time averages of the eigenvalues of $F^{\alpha}{ }_{\beta}$ are all positive. Since we are evolving in the negative time direction, this should lead (through equation (13)) to an exponential decrease in $E_{\alpha}{ }^{i}$. However, since all spatial derivatives appear in the equations in the form $\partial_{\alpha}=E_{\alpha}{ }^{i} \partial_{i}$ we would expect the spatial derivatives to become negligible. That is, the approach to the singularity is local. Furthermore, this positivity of the time averages of the eigenvalues of $F^{\alpha}{ }_{\beta}$ should lead (through equations (14 15) ) to exponential decrease in $r_{\alpha}$ and $A^{\alpha}$. A similar argument applied to equations (18) and (24) and using equations (26) and (28) indicates that as the singularity is approached $Q_{\alpha}$ should become negligible, but $\Omega$ should not, and therefore that $v_{\alpha}$ should become negligible. Thus, as the singularity is approached, the system should be well described by a simplified set of evolution and constraint equations where spatial derivatives as well as $r_{\alpha}, A^{\alpha}$ and $v_{\alpha}$ are negligible. Note that the fact that spatial derivatives are becoming negligible does not mean that the spacetime is becoming homogeneous. Rather the considerable spatial variation is becoming a negligible part of the equations of motion since all spatial derivatives appear multiplied by $E_{\alpha}{ }^{i}$ which is becoming negligible. We now write down this simplified system of evolution and constraint equations where all these terms are neglected. In this limit equation (27) implies that the matricies $\Sigma_{\beta}^{\alpha}$ and $N^{\alpha}{ }_{\beta}$ commute and therefore have the same eigenvalues. The evolution equations for $\Sigma^{\alpha \beta}$ and $N^{\alpha \beta}$ can then be written as evolution equations for their eigenvalues. The non-trivial evolution and constraint equations then become in this limit

$$
\begin{aligned}
\partial_{t} \Sigma_{i} & =(q-2) \Sigma_{i}-2 N_{i}^{2}+\left(\sum_{k=1}^{3} N_{k}\right) N_{i}+\frac{2}{3} Y \\
\partial_{t} N_{i} & =\left(q+2 \Sigma_{i}\right) N_{i} \\
\partial_{t} \Omega & =2(q-2) \Omega
\end{aligned}
$$




$$
\begin{aligned}
& 0=Y+\sum_{k=1}^{3} \Sigma_{k}^{2}+6 \Omega-6 \\
& 0=\sum_{k=1}^{3} \Sigma_{k}^{2}+6 \Omega-3 q
\end{aligned}
$$

Here $\Sigma_{i}$ and $N_{i}$ are the eigenvalues of $\Sigma^{\alpha}{ }_{\beta}$ and $N_{\beta}^{\alpha}$ respectively and $Y$ is given by

$$
Y=\sum_{k=1}^{3} N_{k}^{2}-\frac{1}{2}\left(\sum_{k=1}^{3} N_{k}\right)^{2}
$$

and indicies are not summed over unless explicitly indicated.

Suppose that the dynamics is in a period (called a Kasner epoch) when all the $N_{i}$ are negligibly small. Then it follows from equations (47) and (48) that $q=2$ and therefore, from equations (44) and (46) that $\Omega$ and the $\Sigma_{i}$ are constant. From equations (47) and (45) it follows that there are two possibilities for a Kasner epoch: (i) all the $\Sigma_{i}$ are $\geq-1$ in which case the $N_{i}$ remain negligible and the Kasner epoch lasts all the way to the singularity. (ii) one of the $\Sigma_{i}$ is $<-1$ in which case the corresponding $N_{i}$ grows until it is large enough to bring the Kasner epoch to an end. We now look in more detail at possibility (ii). Let $\Sigma_{1}$ be the $\Sigma_{i}$ that is $<-1$. Then $N_{1}$ is the $N_{i}$ that is growing. We are therefore led to examine equations (44.48) neglecting $N_{2}$ and $N_{3}$ but not $N_{1}$. In this regime equations (44) and (45) become

$$
\begin{array}{r}
\partial_{t} \Sigma_{1}=-S^{2}\left(\Sigma_{1}+4\right) \\
\partial_{t} \Sigma_{2}=-S^{2}\left(\Sigma_{2}-2\right) \\
\partial_{t} \Sigma_{3}=-S^{2}\left(\Sigma_{3}-2\right) \\
\partial_{t} \Omega=-2 S^{2} \Omega
\end{array}
$$

where $S \equiv N_{1} / \sqrt{6}$. Define $Z \equiv 4+\Sigma_{1}$. Then equation (50) becomes $\partial_{t} Z=-S^{2} Z$ while from equations (51) and (52) it follows that there are constants $c_{2}$ and $c_{3}$ with $c_{2}+c_{3}=-1$ such that $\Sigma_{2}=2+c_{2} Z$ and $\Sigma_{3}=2+c_{3} Z$. Similarly, it follows from equation (53) that there is a constant $c_{4}$ such that $\Omega=c_{4} Z^{2}$. Finally, it then follows from equation (47) that $Z$ satisfies the equation of motion

$$
\partial_{t} Z=\left[\frac{1}{6}\left(4-\alpha^{2}\right) Z^{2}-4 Z+6\right] Z
$$

where $\alpha^{2}=1-\left[12 c_{4}+\left(c_{2}-c_{3}\right)^{2}\right]$. Note that the quantity in square brackets vanishes at $Z_{+}$and $Z_{-}$where $Z_{ \pm}=6 /(2 \mp \alpha)$. Therefore the dynamics is a "bounce" from a Kasner epoch corresponding to $Z_{-}$to one corresponding to $Z_{+}$. Use a minus subscript to denote a quantity before the bounce and a plus subscript to denote a quantity after the bounce. We then have $\Omega_{+} / \Omega_{-}=\left(Z_{+} / Z_{-}\right)^{2}$. However, from the definition of $Z$ it follows that $Z_{-}=4+\Sigma_{1-}$ while from the definition of $Z_{ \pm}$it follows that $\left(1 / Z_{+}\right)+\left(1 / Z_{-}\right)=2 / 3$. We then find the following "bounce rule" relating a quantity after the bounce to quantities before the bounce.

$$
\Omega_{+}=\Omega_{-}\left(\frac{3}{5+2 \Sigma_{1-}}\right)^{2}
$$

Note that from the bounce rule it follows that $\Omega$ increases at each bounce. Furthermore, it follows from equation (47) (and from the fact that $\Sigma_{\alpha \beta}$ is trace-free) that the minimum possible value for a $\Sigma_{i}$ during a Kasner epoch is $-2 \sqrt{1-\Omega}$ and therefore that no further bounces can happen once $\Omega>3 / 4$ (though bounces may cease at lower values of $\Omega$ ). Thus we expect that at each spatial point there is a last bounce followed by a Kasner epoch that lasts all the way to the singularity. In other words, we expect the approach to the singularity to be of the Andersson and Rendall class.

\section{RESULTS}

All runs were done in double precision on a SunBlade 2000 with $n=50$ (except for a convergence test which also used $n=25$ ). The equations were evolved from $t=0$ to $t=-90$. For the initial data, the trace of the extrinsic curvature was -1 corresponding to an initial value of $1 / 3$ for $H$. The constants $a_{i}, b_{i}$ and $c_{i}$ characterizing the initial data were

$$
\begin{array}{r}
a_{i}=(0.2,0.1,0.04) \\
b_{i}=(1.7,0.1,0) \\
c_{i}=(0.005,0.005,0.005)
\end{array}
$$

and the constant $c_{0}$ was 0.02 .

We would like to know whether $E_{\alpha}{ }^{i}, r_{\alpha}, A^{\alpha}$ and $v^{\alpha}$ become negligible as the singularity is approached. In figure 1 are plotted the maximum values (over all space, $\alpha$ and $i)$ of $\ln \left|E_{\alpha}{ }^{i}\right|, \ln \left|r_{\alpha}\right|, \ln \left|A^{\alpha}\right|$ and $\ln \left|v^{\alpha}\right|$. Note that after a certain amount of evolution, all these quantities steadily decrease. This indicates that after a certain amount of time spatial derivatives become negligible in the eqautions of motion and that the approximation considered at the end of the previous section becomes valid.

It then follows that the interesting part of the dynamics is the development of the variables at a single point as a function of time. We now present data of that form. The behavior at the spatial point chosen is typical.

The results of a convergence test are plotted in figure 2. Here what is plotted is $4 \mathcal{C}_{q}$ for the $n=50$ run (solid line) and $\mathcal{C}_{q}$ for the $n=25$ run (dotted line). Both quantities are plotted $v s-t$. Note that the two curves roughly agree in magnitude but become out of sync in time. This indicates second order convergence but with the system having sensitive dependence on initial conditions. Similar results were obtained for the other constraints.

Figures 3 and 4 show respectively the diagonal components of $\Sigma_{\alpha \beta}$ and $N_{\alpha \beta}$ in the asymptotic frame, i.e. the frame of the eigenvectors that $\Sigma_{\beta}^{\alpha}$ has at the end of 


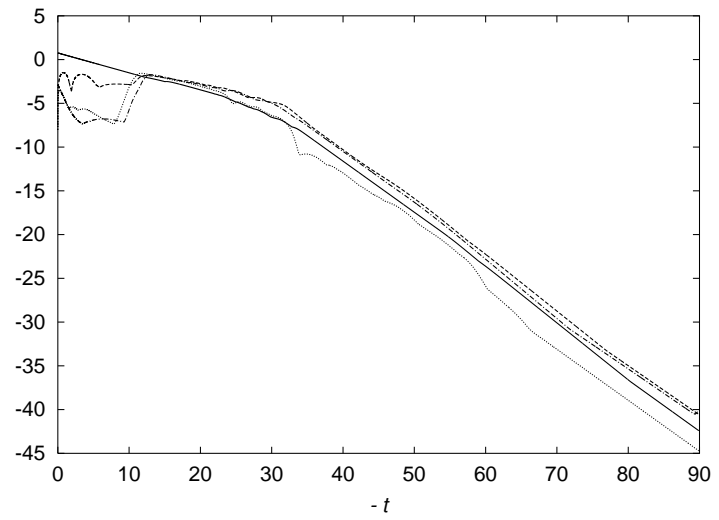

FIG. 1: maximum values of $\ln \left|E_{\alpha}{ }^{i}\right|$ (solid line), $\ln \left|r_{\alpha}\right|$ (dotted line) $\ln \left|A^{\alpha}\right|$ (dot-dashed line) and $\ln \left|v^{\alpha}\right|$ (dashed line) vs $-t$

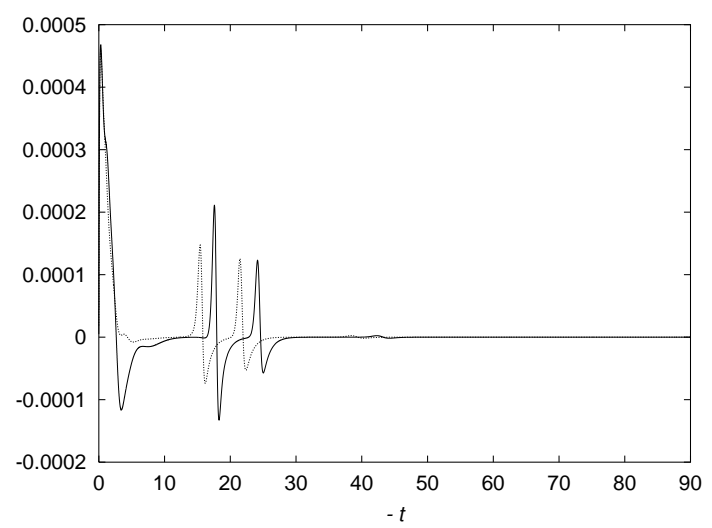

FIG. 2: $4 \mathcal{C}_{q} v s-t$ for the $n=50$ run (solid line) and $\mathcal{C}_{q}$ vs $-t$ for the $n=25$ run (dotted line)

the evolution. For that part of the evolution where the approximation made at the end of the previous section is valid, these diagonal components are the eigenvalues of $\Sigma^{\alpha}{ }_{\beta}$ and $N^{\alpha}{ }_{\beta}$ respectively. Note that the dynamics of the eigenvalues of $\Sigma^{\alpha}{ }_{\beta}$ consists of epochs where they are apporoximately constant (Kasner epochs) punctuated by short bounces. Furthermore the components of $N_{\alpha \beta}$ are negligible except at the bounces. Also note that there is a last bounce and that this coincides with the most negative eigenvalue of $\Sigma^{\alpha}{ }_{\beta}$ becoming greater than -1 .

In figure 5 is plotted $\Omega v s-t$. Note that the behavior of $\Omega$ is a sequence of constant values that are punctuated by short bounces and that the bounces in $\Omega$ occur at the same times as the bounces in $\Sigma_{\alpha \beta}$. The sequence of the values of $\Omega$ is $0.05956,0.1607,0.4139,0.6231$, while the corresponding values of the most negative eigenvalues of $\Sigma_{\beta}^{\alpha}$ are $-1.583,-1.565,-1.277,-0.6596$. These values obey the "bounce rule" of equation (55).

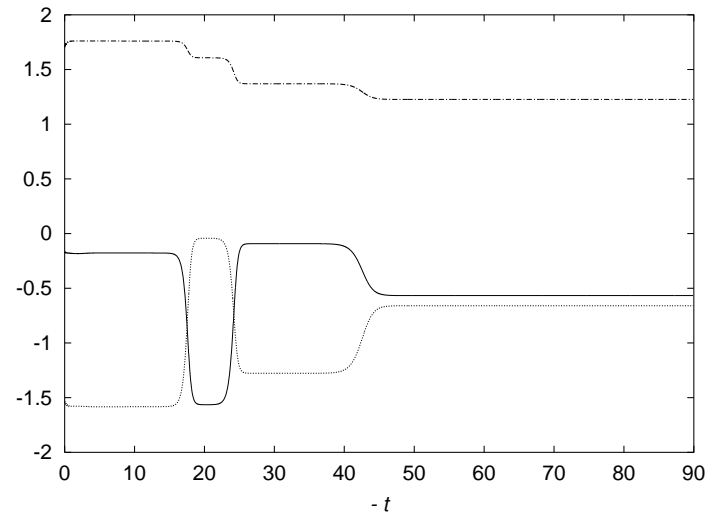

FIG. 3: components of $\Sigma_{\alpha \beta}$ in the asymptotic frame $v s-t$ $\Sigma_{1}$ (solid line), $\Sigma_{2}$ (dotted line) and $\Sigma_{3}$ (dot-dashed line)

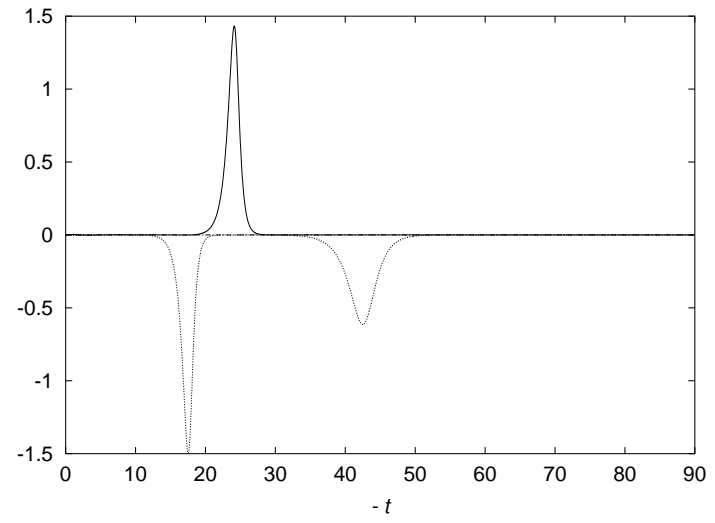

FIG. 4: components of $N_{\alpha \beta}$ in the asymptotic frame $v s-t$ $N_{1}$ (solid line), $N_{2}$ (dotted line) and $N_{3}$ (dot-dashed line)

\section{CONCLUSIONS}

These simulations support the expected picture for the approach to the generic singularity in a spacetime where the matter is a stiff fluid. As the singularity is approached

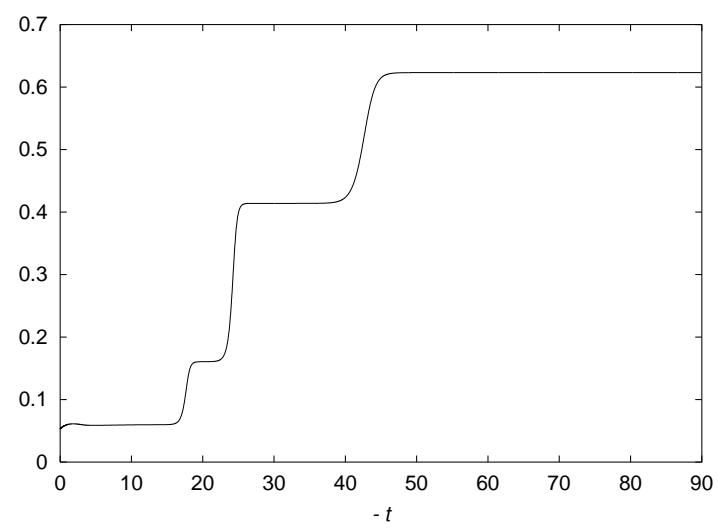

FIG. 5: $\Omega v s-t$ 
the terms in the equations of motion involving spatial derivatives become negligible. The dynamics at each spatial point consists of a sequence of Kasner epochs punctuated by short bounces. The sequence of values of $\Omega$ obeys the expected bounce rule. There is a last bounce, after which the dynamics is described by a single Kasner epoch all the way to the singularity, thus yielding a spacetime in the class of reference 3 ].

What remains to be done is to treat the approach to the singularity for non-stiff fluids. Here the behavior that is expected is quite different. The BKL conjecture is that the matter will become negligible and the dynamics of the gravitational variables as the singularity is approached will be that of vacuum spacetimes. The formalism of 7] includes a class of non-stiff fluids, and the resulting equations are similar to those of the stiff fluid case. Nonetheless, the numerical methods of this paper are not adequate to treat the case of non-stiff fluids. That is because in non-stiff fluids shock waves form, while the numerical methods of the present paper are appropriate for smooth solutions. A shock capturing method would be appropriate to treat the non-stiff fluid case.

\section{ACKNOWLEDGEMENTS}

This work was partially supported by a grant from the National Science and Engineering Research Council of Canada and by NSF grant PHY-0456655 to Oakland University.
[1] for a review see A. Rendall, "Theorems on existence and global dynamics for the Einstein equations" Living Reviews in Relativity (2002-6)

[2] for a review see B. Berger, "Numerical Approaches to spacetime singularities" Living Reviews in Relativity (2002-1)

[3] L. Andersson and A. Rendall, Commun. Math. Phys. 218, 479 (2001)

[4] D. Garfinkle, Phys. Rev. D65, 044029 (2002)

[5] D. Garfinkle, Phys. Rev. Lett. 93, 161101 (2004)

[6] V. Belinskii, I. Khalatnikov and E. Lifschitz, Sov. Phys. Usp. 13, 745 (1971)
[7] C. Uggla, H. van Elst, J. Wainwright and G.F.R. Ellis, Phys. Rev. D68, 103502 (2003)

[8] J.W. York, Phys. Rev. Lett. 26, 1656 (1971)

[9] J. Isenberg, Class. Quant. Grav. 12, 2249 (1995)

[10] W. Press, S. Teukolsky, W. Vetterling and B. Flannery, Numerical Recipes in FORTRAN, second edition (Cambridge University Press, Cambridge, 1992)

[11] D. Garfinkle and C. Gundlach, gr-qc/0501031

[12] M. Choptuik, in Deterministic Chaos in General Relativity, edited by D. Hobill, A. Burd and A. Coley (Plenum, New York, 1994), pp. 155-175 